\title{
FAMILIES OF PRINCIPAL SOLUTIONS OF ORDINARY DIFFERENTIAL EQUATIONS(1)
}

\author{
BY \\ ERLING WILLIAM CHAMBERLAIN
}

\section{PART I. INTRODUCTION}

Let $A\left(y, D y, \cdots, D^{n} y\right)=0$ be an algebraic differential equation belonging to Strodt's class (D) [1, p. 5], and let $M$ be a principal monomial $[1, \S 66]$ for $A=0$. In search for principal solutions of $A=0$ (i.e., solutions which are $\sim M$ ) one substitutes $y=M(1+z)$. This almost always [2, $\$ 121]$ leads to a differential equation $P(z)=0$ in which $P$ is an asymptotically quasilinear algebraic differential operator having a nonexceptional factorization sequence $\left(W_{1}, \cdots, W_{n}\right)$ such that $P$ is normal with respect to $\left(W_{1}, \cdots, W_{n}, r\right)$ for a sufficiently large positive integer $r$. Strodt [2] has shown that $P=0$ has at least one solution $Z \prec 1$, and accordingly $A=0$ has at least one solution $Y=M(1+Z) \sim M$; but if $P$ is, in addition, uniformly quasilinear, then $P=0$ has a $u$-parameter family of solutions $\prec 1$ (and $A=0$ has a $u$-parameter family of solutions $\sim M$ ), where $u$ is the number of indices $i$ for which $[I F]^{\sim}\left(W_{i}, \phi\right)<0(\phi$ denoting the direction in which the complex variable approaches infinity). The significance of the number $u$ in the more general a.q.l. situation has hitherto been obscured by the complicated manipulations used to ascertain the mere existence of a solution $\prec 1$ for a.q.1. equations.

In the present study, a $u$-parameter family of solutions $\prec 1$ is exhibited for a large class of normal a.q.l. equations $P=0$. We require that the factorization sequence for $P$ satisfy a condition which resembles (and includes) the classical "distinctness of characteristic roots" in the case of linear differential equations with constant coefficients ( $\$ 2$, below). The virtue of this condition is that it allows us to pass from approximate factorizations of linear operators in terms of the operators $\dot{W}_{i}=\left(1-W_{i}^{-1} D\right)$ to exact factorizations in terms of certain $\dot{V}_{i}$ such that $V_{i} \sim W_{i}$. Once the linear part of an algebraic a.q.l. equation has been exactly factored, the situation may be said to be well in hand. Among other things, exact factorization enables us to measure with considerable precision the asymptotic size of our small $(\prec 1)$ solutions, and even to obtain a sort of asymptotic development for them. Other phenomena used to advantage in this study include the invariance of normality and asymptotic quasilinearity of differential operators

Received by the editors November 2, 1961 .

(1) This research was supported by Air Force Contract No. AF 49(638)-644 while at Columbia University. 
under small displacements of dependent variable or small displacements of factorization sequences, and the evolution of new a.q.l. operators from old ones under judicious substitutions.

For an index of terminology used in references [1] and [2] and in the present paper, the reader is referred to [2, Part IX, pp. 105-107].

\section{PART II. EXACT FACTORIZATION OF LINEAR DIFFERENTIAL OPERATORS}

1. LEMMA (APPROXIMATE FACTORIZATION $\rightarrow$ PARTLY EXACT FACTORIZATION). Let $W_{1}, \cdots, W_{n}$ be logarithmic monomials in the divergence class. Let $W_{i}-W_{1} \approx W_{i}$ for $i=2,3, \cdots, n$. Let $U_{i}=W_{i}-W_{1}(i=2,3, \cdots, n)$ and let $\left(U_{2}, U_{3}, \cdots, U_{n}\right)$ be unblocked in $(\alpha, \phi, \beta)$. Let $L=\dot{W}_{n} \cdots \dot{W}_{1}+\sum_{i=1}^{n} E_{i} \dot{W}_{i} \cdots \dot{W}_{1}+E_{0}$, with each $E_{i} \prec 1$ in $F(\alpha, \beta)$. Then there exists a function $V_{1}$ such that $V_{1} \sim W_{1}$ and such that $L=\dot{W}_{n} \cdots W_{2} \dot{V}_{1}+\sum_{i=1}^{n} E_{1 i} \dot{W}_{i} \cdots \dot{W}_{2} \dot{V}_{1}$ with each $E_{1 i} \prec 1$ (all relations holding in $F(\alpha, \beta)$ ).

Proof. In the notation of $[2, \S 4]$, we want $R^{*} L\left(V_{1}\right)=0$. According to [2, $\S \S 32,33]$, we have $R^{*} L(y)=\ddot{W}_{n}(y) \cdots \ddot{W}_{1}(y) \cdot 1+\sum_{i=1}^{n} E_{i} \ddot{W}_{i}(y) \cdots \ddot{W}_{1}(y) \cdot 1+E_{0}$, where $\ddot{W}(y)=\left(1-W^{-1} y-W^{-1} D\right)$. Under the substitution $y=W_{1}(1-z)$ we have $\ddot{W}_{i}(y)=U_{i} W_{i}^{-1}\left(\dot{U}_{i}+W_{1} U_{i}^{-1} z\right)$, where $U_{i} W_{i}^{-1} \approx 1$ and $W_{1} U_{i}^{-1} \precsim 1$ for all relevant $i$. One proves by induction on $i$ that $\ddot{W}_{i}(y) \cdots \ddot{W}_{1}(y) \cdot 1=b_{i} B_{i}(z)$, where $b_{i} \approx 1$ and $B_{i}$ is a differential operator which is $\sim(s) \dot{U}(i, \cdots, 2)$. In carrying out this proof, it is convenient to dispose of terms of the form

$$
\dot{U}_{j}\left\{c z^{t_{0}}\left(\dot{U}_{2} z\right)^{t_{1}} \cdots\left(\dot{U}_{h} \dot{U}_{h-1} \cdots \dot{U}_{2} z\right)^{t_{h-1}}\right\}
$$

$(h<j)$ by means of the relations $\dot{U}(A B)=A \dot{U}(B)+B \dot{U}(A)-A B$ and

$$
\dot{U}_{j}\left(\dot{U}_{h} \cdots \dot{U}_{2} z\right)=U_{h+1} U_{j}^{-1}\left(\dot{U}_{h+1} \dot{U}_{h} \cdots \dot{U}_{2} z\right)+\left(1-U_{h+1} U_{j}^{-1}\right) \dot{U}_{h} \cdots \dot{U}_{2} z .
$$

Once the properties of $b_{i}$ and $B_{i}$ are established, it follows immediately that $R^{*} L\left(W_{1}(1-z)\right)=b B(z)$, where $b \approx 1$ and $B \sim(s) \dot{U}(n, \cdots, 2)$. Since $\left(U_{2}, \cdots, U_{n}\right)$ is unblocked, Theorem II of [2] implies the existence of a solution $Z$ of $B(z)=0$ such that $Z \prec 1$ in $F(\alpha, \beta)$. Define $V_{1}=W_{1}(1-Z)$. The lemma now follows from the fact that $R^{*} L\left(V_{1}\right)=0$ and $\left(V_{1}, W_{2}, \cdots, W_{n}\right)$ is a factorization sequence for $L[2, \S 54]$.

2. Definition. We shall call $\left(W_{1}, \cdots, W_{n}\right)$ separated when the $W_{i}$ are $\sim$ logarithmic monomials in the divergence class satisfying

(1) $W_{i}-W_{j} \approx W_{i}$ for $i>j$,

(2) each $W_{i}-W_{j}$ is unblocked in $(\alpha, \phi, \beta)$.

THEOREM I (APPROXIMATE FACTORIZATION $\rightarrow$ EXACT FACTORIZATION). Let $\left(W_{1}, \cdots, W_{n}\right)$ be a separated factorization sequence for the linear differential operator $L$. Then there exist functions $V_{1}, \cdots, V_{n}$ such that $V_{i} \sim W_{i}(i=1, \cdots, n)$ and such that $L=u \dot{V}_{n} \cdots \dot{V}_{1}$ for some $u \sim 1$. 
Proof. By Lemma 1, $L=L_{1} \dot{V}_{1}$ for some function $V_{1} \sim W_{1}$ and a certain operator $L_{1}$ such that $L_{1} \sim \dot{W}(n, \cdots, 2)$. It is clear that $L_{1}$ is qualified to play the role which $L$ plays in Lemma 1 , so we get $L_{1}=L_{2} \dot{V}_{2}$ where $V_{2} \sim W_{2}$ and $L_{2} \sim \dot{W}(n, \cdots, 3)$. The conclusion of the present theorem is reached after finitely many repetitions of this same argument.

PART III. ON THE EQUATION $\dot{W}_{n} \dot{W}_{n-1} \cdots \dot{W}_{1} y=0$

3. We present here some results of a computational nature, necessary for the subsequent study of transformations of differential operators under substitutions for the dependent variable.

Lemma. Let $W_{i} \sim$ a logarithmic monomial in the divergence class $(i=1, \cdots, n)$, and $W_{i}-W_{j} \approx W_{i}$ if $i>j$. Let $\delta>\tau>0$. Let $k \in(1, \cdots, n)$. Let

$$
W_{k} \sim c x^{-1}(\log x)^{-1} \cdots\left(\log _{p-1} x\right)^{-1}\left(\log _{p} x\right)^{-1+\tau}\left(\log _{p+1} x\right)^{a_{1}} \cdots\left(\log _{p+s} x\right)^{a_{s}} .
$$

Let $F=\left(\log _{p} x\right)^{-\delta}$. Let $G \prec 1$ and $D G=F W_{k}$ (see Lemma $\zeta$ ). Let $H_{k} \equiv 1$ and $H_{s}=\left(1-W_{k} W_{s}^{-1}\right)^{-1}\left(1-W_{k} W_{s+1}^{-1}\right)^{-1} \cdots\left(1-W_{k} W_{k-1}^{-1}\right)^{-1}$ for $s=k-1, \cdots, 1$. Let $K_{s}=H_{s} \exp \int_{x_{0}} W_{k}(s=1, \cdots, k)$. Let $z$ be an admissible function. Then:

(3.1) $\dot{W}_{s}\left(K_{s}\right)=u_{s} K_{s+1}$, with $u_{s} \sim 1 \quad(s=1, \cdots, k-1)$.

(3.2) $\dot{W}_{s}\left(K_{s} z\right)=u_{s} K_{s+1} \dot{M}_{s} z$, with $M_{s}=u_{s}\left(W_{s}-W_{k}\right) \quad(s=1, \cdots, k-1)$.

(3.3) $\dot{M}_{s}(G) \sim G \quad(s=1, \cdots, k-1)$.

(3.4) $\dot{W}_{s}\left(G K_{s}\right)=v_{s} G K_{s+1}$, with $v_{s} \sim 1 \quad(s=1, \cdots, k-1)$.

(3.5) $\dot{W}_{s}\left(G K_{s} z\right)=v_{s} G K_{s+1} \dot{U}_{s} z$, with $U_{s}=v_{s}\left(W_{s}-W_{k}\right) \quad(s=1, \cdots, k-1)$.

(3.6) For $s=1, \cdots, k-1, \dot{W}_{s} \cdots \dot{W}_{1}\left(K_{1} z\right)=K_{s+1} \Psi_{s} \dot{N}_{s} \cdots \dot{N}_{1} z$, with $\Psi_{s} \sim 1$ and $N_{i} \sim W_{i}-W_{k} \quad(i=1, \cdots, s)$.

(3.7) For $s=1, \cdots, k-1, \dot{W}_{s} \cdots \dot{W}_{1}\left(G K_{1} z\right)=G K_{s+1} \Phi_{s} \dot{V}_{s} \cdots \dot{V}_{1} z$, with $\Phi_{s} \sim 1$ and $V_{i} \sim W_{i}-W_{k} \quad(i=1, \cdots, s)$.

(3.8) $\dot{W}_{k}\left(z \exp \int_{x_{0}} W_{k}\right)=-W_{k}^{-1} D z \exp \int_{x_{0}} W_{k}$.

(3.9) $\dot{W}_{k}\left(z G \exp \int_{x_{0}} W_{k}\right)=-F \exp \int_{x_{0}} W_{k} \dot{Q}$, where $Q=-G^{-1} D G$.

Let $\Phi_{t}=1-W_{k} W_{t}^{-1}-F^{-1} W_{t}^{-1} D F$ and $\Psi_{t}=1-W_{k} W_{t}^{-1}$, for $\left.t=k+1, \cdots, n\right)$. $\Phi_{t}$ and $\Psi_{t}$ are easily seen to be $\approx 1$.

(3.10) $\dot{W}_{t}\left(\exp \int_{x_{0}} W_{k}\right)=\Psi_{t} \exp \int_{x_{0}} W_{k} \quad(t=k+1, \cdots, n)$.

(3.11) $\dot{W}_{t}\left(F \exp \int_{x_{0}} W_{k}\right)=\Phi_{t} F \exp \int_{x_{0}} W_{k} \quad(t=k+1, \cdots, n)$.

(3.12) $\dot{W}_{t}\left(z \exp \int_{x_{0}} W_{k}\right)=\Psi_{t} \dot{M}_{t} z \exp \int_{x_{0}} W_{k}$, where $M_{t}=\Psi_{t} W_{t} \quad(t=k+1, \cdots, n)$.

(3.13) $\dot{W}_{t}\left(z F \exp \int_{x_{0}} W_{k}\right)=\Phi_{t} \dot{U}_{t} z F \exp \int_{x_{0}} W_{k}$, where $U_{t}=\Phi_{t} W_{t} \quad(t=k+1, \cdots, n$.

(3.14) $M_{t} \sim U_{t} \sim W_{t}-W_{k} \quad(t=k+1, \cdots, n)$.

(3.15) $\dot{W}_{t} \cdots \dot{W}_{k+1}\left(z \exp \int_{x_{0}} W_{k}\right)=\Psi_{t}^{*} \exp \int_{x_{0}} W_{k} \dot{N}_{t} \cdots \dot{N}_{k+1} z$, with $\Psi_{t}^{*} \approx 1$ and $N_{t} \sim W_{t}-W_{k} \quad(t=k+1, \cdots, n)$.

(3.16) $\dot{W}_{t} \cdots \dot{W}_{k+1}\left(z F \exp \int_{x_{0}} W_{k}\right)=\Phi_{t}^{*} F \exp \int_{x_{0}} W_{k} \dot{V}_{t} \cdots \dot{V}_{k+1} z$, with $\Phi_{t}^{*} \approx 1$ and $V_{t} \sim W_{t}-W_{k} \quad(t=k+1, \cdots, n)$.

Proof. In verifying (3.1)-(3.16), much use may be made of the relation (R): $\dot{W}(T y)=(\dot{W}(T))(\dot{M}(y))$, where $M=W T^{-1} \dot{W}(T)$ (cf. [2, $\left.\left.\$ 113\right]\right)$. 
For (3.1): $\dot{W}_{s}\left(K_{s}\right)=H_{s}\left(1-W_{s}^{-1}\left(H_{s}^{-1} D H_{s}+W_{k}\right)\right) \exp \int_{x_{0}} W_{k} . H_{s}^{-1} D H_{s} \prec W_{k}$ because $\delta_{i}\left(H_{s}\right)=0(i=0,1, \cdots, p-1)$, implying $\delta_{i}\left(H_{s}{ }^{-1} D H_{s}\right)=-1(i=0, \cdots, p)$. Hence $1-W_{s}^{-1}\left(H_{s}^{-1} D H_{s}+W_{k}\right) \sim 1-W_{s}^{-1} W_{k}$, and the conclusion follows because $\left(1-W_{s}^{-1} W_{k}\right) H_{s}=H_{s+1}$.

For (3.2): In applying (R) and (3.1), we have $M_{s}=W_{s} u_{s} K_{s+1} K_{s}^{-1}=u_{s}\left(W_{s}-W_{k}\right)$.

For (3.3): The conditions imply $G \sim(\tau-\delta)^{-1} x(\log x) \cdots\left(\log _{p} x\right) F W_{k}$. We also have $M_{s} \approx W_{k}$; therefore $M_{s}^{-1} D G \approx F \prec G$.

For (3.4): Apply (3.2) with $z$ replaced by $G$.

For (3.5): Apply (R) together with (3.4).

For (3.6): For successive values of $s$, we show that if $\Psi_{s-1} \sim 1$, then $\dot{W}_{s}\left(\Psi_{s-1} K_{s} z^{\prime}\right)=K_{s+1} \Psi_{s} \dot{N}_{s} z^{\prime}$ where $z^{\prime}=\dot{N}_{s-1} \cdots \dot{N}_{1} z, \Psi_{s} \sim 1$, and $N_{s} \sim W_{s}-W_{k}$. By (R), $\dot{W}_{s}\left(\Psi_{s-1} K_{s} z^{\prime}\right)=\left(\dot{W}_{s} \Psi_{s-1}\right)\left(\dot{S}\left(K_{s} z^{\prime}\right)\right)$, where $\dot{W}_{s} \Psi_{s-1} \sim 1$ because $\Psi_{s-1} \sim 1$ while $S=v W_{s}$, where $v=\Psi_{s-1}^{-1} \dot{W}_{s}\left(\Psi_{s-1}\right)$ which is $\sim 1$. Then $\dot{S}\left(K_{s} z^{\prime}\right)=(1 / v) \dot{W}_{s}\left(K_{s} z^{\prime}\right)$ $+(1-1 / v) K_{s} z^{\prime}=(1 / v)\left(u_{s} K_{s+1} \dot{M}_{s} z^{\prime}+(v-1) K_{s} z^{\prime}\right)$, using (3.2), = $K_{s+1}\left(u_{s} / v+((v-1) / v)\left(K_{s} / K_{s+1}\right)\right)\left(z^{\prime}-D z^{\prime} / M_{s}\left(1+(v-1) K_{s} / u_{s} K_{s+1}\right)\right)$. To obtain the desired result we set $N_{s}=M_{s}\left(1+(v-1) K_{s} / u_{s} K_{s+1}\right)$ and $\Psi_{s}=\left(\dot{W}_{s}\left(\Psi_{s-1}\right)\right)\left(u_{s} / v+((v-1) / v)\left(K / K_{s+1}\right)\right)$. Noting that $K_{s+1} \succsim K_{s}$ and $(v-1) v \prec 1$, we have $\Psi_{s} \sim 1$ and $N_{s} \sim M_{s} \sim W_{s}-W_{k}$ as claimed. To begin the induction, (3.2) may be applied for the case $s=1$.

For (3.7): Apply (R) and (3.5) repeatedly.

(3.8)-(3.11) follow from easy computations.

For (3.12) and (3.13): Apply (R), (3.10), and (3.11).

(3.14) is obvious.

For (3.15) and (3.16): Apply (R), (3.12), and (3.13) repeatedly.

4. THEOREM II. Let the hypotheses and notation of Lemma 3 be in force. Let $-\pi \leqq \alpha<\phi<\beta \leqq \pi$. Let $W_{1}-W_{k}, W_{2}-W_{k}, \cdots, W_{k-1}-W_{k}$ be unblocked in $(\alpha, \phi, \beta)$. Let $S$ be an element of $F(\alpha, \beta)$ in which $W_{k}$ is analytic, and let $x_{0} \in S$. Then for every complex number $g \neq 0$, the differential equation

$$
\dot{W}_{n} \cdots \dot{W}_{1} y=0
$$

has a solution $y_{g}$ which is $\sim g H_{1} \exp \int_{x_{0}} W_{k}$.

Proof. Let $y=g H_{1} \exp \int_{x_{0}} W_{k} z$. (4.1) becomes

$$
\dot{W}_{n} \cdots \dot{W}_{k}\left(\Psi_{k-1} \exp \int_{x_{0}} W_{k} \dot{N}_{k-1} \cdots \dot{N}_{1} z\right)=0
$$

(cf. (3.6)), or $\dot{W}_{n} \cdots \dot{W}_{k+1}\left(-W_{k}^{-1} \exp \int_{x_{0}} W_{k} D\left(\Psi_{k-1} \dot{N}_{k-1} \cdots \dot{N}_{1} z\right)\right)=0$ (cf. (3.8)), and hence it suffices to solve the equation $\dot{N}_{k-1} \cdots \dot{N}_{1} z=\Psi_{k-1}^{-1}$ for a solution $z \sim 1$. Setting $z=1+w$, we seek a function $w \prec 1$ such that $\dot{N}_{k-1} \cdots \dot{N}_{1} w=\Psi_{k-1}^{-1}-1$. But $\left(N_{1}, \cdots, N_{k-1}\right)$ is unblocked in $(\alpha, \phi, \beta)$ and $\Psi_{k-1}^{-1}-1 \prec 1$ in $F(\alpha, \beta)$, so the existence of a solution $w^{*} \prec 1$ may be inferred from $[2, \S 114]$. (A direct proof using $[2, \S 78]$ is also feasible: There exists a 
$w_{k-1} \prec 1$ such that $\dot{N}_{k-1} w_{k-1}=\Psi_{k-1}^{-1}-1$, there exists a $w_{k-2} \prec 1$ such that $\dot{N}_{k-2} w_{k-2}=w_{k-1}, \cdots$, and finally there exists a $w_{1}=w^{*} \prec 1$ such that $\dot{N}_{1} w_{1}=w_{2}$.) Taking $y_{g}=g H_{1}\left(1+w^{*}\right) \exp \int_{x_{0}} W_{k}$, it is clear that the conclusions of the theorem are satisfied.

5. Lemma. Let the hypotheses and notation of 4 . be in force. Let $y=y_{g}+G K_{1} z$. Then

$$
\begin{aligned}
& \dot{W}_{s} \cdots \dot{W}_{1} y=\dot{W}_{s} \cdots \dot{W}_{1} y_{g}+G K_{s+1} \Phi_{s} \dot{V}_{s} \cdots \dot{V}_{1} z \quad(s=1, \cdots, k-1) \\
& \dot{W}_{k} \cdots \dot{W}_{1} y=\left(-F \bar{\Phi}_{k-1} \exp \int_{x_{0}} W_{k}\right) \dot{Q}^{\prime} \dot{V}_{k-1} \cdots \dot{V}_{1} z \text {, with } \bar{\Phi}_{k-1} \sim 1 ; \text { and } \\
& \dot{W}_{t} \cdots \dot{W}_{1} y=\left(-F \bar{\Phi}_{t} \exp \int_{x_{0}} W_{k}\right) \dot{V}_{t}^{\prime} \cdots \dot{V}_{k+1}^{\prime} \dot{Q}^{\prime} \dot{V}_{k-1} \cdots \dot{V}_{1} z, \text { with } \bar{\Phi}_{t} \approx 1, Q^{\prime} \sim Q,
\end{aligned}
$$
and $V_{t}^{\prime} \sim V_{t} \quad(t=k+1, \cdots, n)$.

Proof. Clearly $\dot{W}_{s} \cdots \dot{W}_{1} y_{g}=0$ for $s=k, \cdots, n$, and

$$
\dot{W}_{s} \cdots \dot{W}_{1}\left(G K_{1} z\right)=G K_{s+1} \Phi_{s} \dot{V}_{s} \cdots \dot{V}_{1} z
$$

with $\Phi_{s}$ and the $V$ 's as in (3.7), for $s=1, \cdots, k-1$.

$\dot{W}_{k} \cdots \dot{W}_{1}\left(G K_{1} z\right)=\dot{W}_{k}\left(G \exp \int_{x_{0}} W_{k} \Phi_{k-1} \dot{V}_{k-1} \cdots \dot{V}_{1} z\right)$ and we use (3.9) with $\left(\Phi_{k-1} \dot{V}_{k-1} \cdots \dot{V}_{1} z\right)$ in place of the $z$ in (3.9). This gives

$$
-F \exp \int_{x_{0}} W_{k}\left\{\dot{Q}\left(\Phi_{k-1} \dot{V}_{k-1} \cdots \dot{V}_{1} z\right)\right\} .
$$

On $\{\cdots\}$ we use (R) with $T=\Phi_{k-1}$ and $y=\dot{V}_{k-1} \cdots \dot{V}_{1} z$ (see proof of Lemma 3), which gives $\dot{W}_{k} \cdots \dot{W}_{1}\left(G K_{1} z\right)=\left(-F \bar{\Phi}_{k-1} \exp \int_{x_{0}} W_{k}\right) \dot{Q}^{\prime} \dot{V}_{k-1} \cdots \dot{V}_{1} z$, where $\bar{\Phi}_{k-1}=\dot{Q}\left(\Phi_{k-1}\right)$ and $Q^{\prime}=Q \Phi_{k-1}^{-1} \bar{\Phi}_{k-1}$.

If $t=k+1, \cdots, n$ we have

$$
\dot{W}_{t} \cdots \dot{W}_{1}\left(G K_{1} z\right)=\dot{W}_{t} \cdots \dot{W}_{k+1}\left(-F \exp \int_{x_{0}} W_{k} \bar{\Phi}_{k-1} \dot{Q}^{\prime} \dot{V}_{k-1} \cdots \dot{V}_{1} z\right),
$$

and we use (3.16) with $z$ replaced by $\bar{\Phi}_{k-1} \dot{Q}^{\prime} \dot{V}_{k-1} \cdots \dot{V}_{1} z$. This gives $\dot{W}_{t} \cdots \dot{W}_{1}\left(G K_{1} z\right)$ $=-F \exp \int_{x_{0}} W_{k} \Phi_{t}^{*}\left\{\dot{V}_{t} \cdots \dot{V}_{k+1}\left(\bar{\Phi}_{k-1} \dot{Q}^{\prime} \dot{V}_{k-1} \cdots \dot{V}_{1} z\right)\right\}$. On $\{\cdots\}$ we use (R) as in the previous paragraph to "permute" $\bar{\Phi}_{k-1}$ past $\dot{V}_{t} \cdots \dot{V}_{k+1}$ and obtain the stated result.

\section{PART IV. SOlUtions OF NONLINEAR EQUATIONS}

6. Definition. Let $P \sim(w) \dot{W}(n, \cdots, 1)$. We say that $P$ is condensed with respect to $\left(W_{1}, \cdots, W_{n}\right)$, and write $P \sim(c, w) \dot{W}(n, \cdots, 1)$, if $[H L P]^{\sim}=u \dot{W}_{n} \cdots \dot{W}_{1}$ for some $u \sim 1$. If $P \sim(c, w) \dot{W}(n, \cdots, 1)$ and $P$ is also normal with respect to $\left(W_{1}, \cdots, W_{n}, r\right)[2, \S 102]$, we say that $P$ is condensed with respect to $\left(W_{1}, \cdots, W_{n}, r\right)$ and write $P \sim(r, c, w) \dot{W}(n, \cdots, 1)$.

7. Lemma. Let $\left(W_{1}, \cdots, W_{n}\right)$ be separated and unblocked in $(\alpha, \phi, \beta)$. Let $P^{\prime}$ be normal with respect to $\left(W_{1}, \cdots, W_{n}, r\right)$. Then there exists a function $Y \prec 1$ and functions $V_{1}, \cdots, V_{n}$ with $W_{i} \sim V_{i}(i=1, \cdots, n)$ such that if $P(y)=P^{\prime}(Y+y)$, then $P \sim(r, c, w) \dot{V}(n, \cdots, 1)$ and $P(0)=0$. 
Proof. By Theorem IIIa of [2], there exists a solution of $P^{\prime}(y)=0$ which is $\prec 1$. Let $Y$ be one such solution, and let $P(y)=P^{\prime}(Y+y)$. Then $P(0)=0$.

By Lemma $\alpha, P$ is normal with respect to $\left(W_{1}, \cdots, W_{n}, r\right)$. Theorem $I$ above shows that the approximate factorization of $[H L P]^{\sim}$ using $\left(W_{1}, \cdots, W_{n}\right)$ can be converted into an exact factorization in terms of $\left(V_{1}, \cdots, V_{n}\right)$ where $V_{i} \sim W_{i}$ for all $i$. Lemma $\beta$ shows that $P$ is normal with respect to $\left(V_{1}, \cdots, V_{n}, r\right)$. Hence $P \sim(r, c, w) \dot{V}(n, \cdots, 1)$.

8. LEMMA. Let the hypotheses and notation of Lemma 5 be in force. Let $[I F]^{\sim}\left(W_{k}, \phi\right)<0$. Let $X_{i}=V_{i}(i=1, \cdots, k-1), \quad X_{k}=Q^{\prime}$, and $X_{i}=V_{i}^{\prime} \quad(i=$ $k+1, \cdots, n)$. Let $P \sim(r, c, w) \dot{W}(n, \cdots, 1)$, and let $R\left(z, \dot{X}_{1} z, \cdots, \dot{X}_{n} \cdots \dot{X}_{1} z\right)$ $=P\left(y, \dot{W}_{1} y, \cdots, \dot{W}_{n} \cdots \dot{W}_{1} y\right)$. Then whenever $z \prec 1, \quad R\left(z, \dot{X}_{1} z, \cdots, \dot{X}_{n} \cdots \dot{X}_{1} z\right)$ $\prec F \exp \int_{x_{0}} W_{k}$.

Proof. Since $\dot{W}_{n} \cdots \dot{W}_{1}\left(y_{g}\right)=0$, and since $\dot{X}_{n} \cdots \dot{X}_{1}$ is unimajoral, Lemma 5 immediately implies $\dot{W}_{n} \cdots \dot{W}_{1} y \prec F \exp \int_{x_{0}} W_{k}$ for every $z \prec 1$. Let $\theta=\theta_{r}$. (We assume, with no loss of generality, that $r \geqq p+1$.) Then $P(y)-[H L P]^{\sim}(y)$ may be expressed as a polynomial in $y, \theta y, \cdots, \theta^{n} y$ such that each monomial has degree $\geqq 2$ and each coefficient is $\precsim 1$. It is easily shown that for every $z \prec 1$. $P(y)-[H L P]^{\sim}(y) \precsim\left(\theta^{t} y\right)^{2}$ for some $t \in(0, \cdots, n)$. In fact, for the functions $y$ being considered, it is readily verified that $\theta^{t} y=E_{t} J_{t} \exp \int_{x_{0}} W_{k}$ where $E_{t} \sim 1$ and $J_{t}$ is a logarithmic monomial such that $\delta_{i}\left(J_{t}\right)=0(i=0, \cdots, p-1 ; t=2, \cdots, n)$. Hence there exists a logarithmic monomial $J^{*}$ such that $\delta_{i}\left(J^{*}\right)=0(i=0, \cdots, p-1)$ and such that $J^{*} \exp \int_{x_{0}} 2 W_{k} \succ P\left(y, \dot{W}_{1} y, \cdots, \dot{W}_{n} \cdots \dot{W}_{1} y\right)-[H L P]^{\sim}(y)$ whenever $z \prec 1$. But $J^{*} \exp \int_{x_{0}} 2 W_{k} \prec F \exp \int_{x_{0}} W_{k}$ by Lemma $\varepsilon$. It follows that $R\left(z, \dot{X}_{1} z, \cdots, \dot{X}_{n} \cdots \dot{X}_{1} z\right)$ $\prec F \exp \int_{x_{0}} W_{k}$ whenever $z \prec 1$.

9. LEMMA. Let the hypotheses and notation of Lemma 8 be in force. Let $P_{s}$ and $R_{s}$ denote the partial derivatives of $P$ and $R$ with respect to their sth arguments. Then $P_{s}\left(y, \dot{W}_{1} y, \cdots, \dot{W}_{n} \cdots \dot{W}_{1} y\right) \prec J^{\prime \prime} \exp \int_{x_{0}} W_{k}(s=0, \cdots, n-1)$, where $J^{\prime \prime}$ is any sufficiently large logarithmic monomial such that $\delta_{i}\left(J^{\prime \prime}\right)=0$ $(i=0, \cdots, p-1)$.

Proof. Let $\bar{P}=P-[H L P]^{\sim}$. Then $\bar{P}_{s}=P_{s}(s=0, \cdots, n-1)$, since $P$ is condensed. We have

$$
\bar{P}\left(y, \dot{W}_{1} y, \cdots, \dot{W}_{n} \cdots \dot{W}_{1} y\right)=\Sigma p_{\hat{\jmath}} y^{j_{0}}(\theta y)^{j_{1}} \cdots\left(\theta^{n} y\right)^{j_{n}}
$$

with $j_{0}+j_{1}+\cdots+j_{n} \geqq 2$ and $j_{t} p_{\hat{j}} \precsim A_{1} \cdots A_{t}(t=1, \cdots, n)$ for each $\hat{j}$, where $A_{t}=-\left(x(\log x) \cdots\left(\log _{r-1} x\right) W_{t}\right)^{-1}$. Also, by $[2, ! \S 100]$, we have

$$
\theta^{t} y=\sum_{h=0}^{t} G_{t h} \dot{W}_{h} \cdots \dot{W}_{1} y
$$

with $G_{t h} \precsim 1 / A_{1} \cdots A_{t}$. Hence

$$
P_{s}\left(y, \dot{W}_{1} y, \cdots, \dot{W}_{n} \cdots \dot{W}_{1} y\right)=\sum p_{\hat{\jmath}} y^{j_{0}}(\theta y)^{j_{1}} \cdots\left(\theta^{n} y\right)^{j_{n}} C_{\hat{\jmath}},
$$


where $C_{j}=j_{s}\left(\theta^{s} y\right)^{-1} G_{s s}+j_{s+1}\left(\theta^{s+1} y\right)^{-1} G_{s+1, s}+\cdots+j_{n}\left(\theta^{n} y\right)^{-1} G_{n s}$. But $j_{s+q} p_{j} G_{s+q, s} \precsim 1(q=0, \cdots, n-s)$. Hence we may write $P_{s}\left(y, \dot{W}_{1} y, \cdots, \dot{W}_{n} \cdots \dot{W}_{1} y\right)$ as a polynomial in $y, \theta y, \cdots, \theta^{n} y$ in which each monomial is of degree $\geqq 1$ and each coefficient is $\precsim 1$. The argument given in proof of Lemma 8 shows that there exists a $J^{\prime \prime}$ with the prescribed properties.

10. LEMma. Let the hypotheses and notation of Lemma 9 be in force. Then $R_{s}\left(z, \dot{X}_{1} z, \cdots, \dot{X}_{n} \cdots \dot{X}_{1} z\right) \prec F \exp \int_{x_{0}} W_{k}$ for every $z \prec 1$ and every $s \in(0,1, \cdots, n-1)$.

Proof. $R_{s}\left(z, \dot{X}_{1} z, \cdots, \dot{X}_{n} \cdots \dot{X}_{1} z\right)=P_{s}\left(y, \dot{W}_{1} y, \cdots, \dot{W}_{n} \cdots \dot{W}_{1} y\right) T(s)$ where $T(0)=G K_{1}, T(s) \sim G K_{s+1}(s=1, \cdots, k-1)$, and $T(s) \approx-F \exp \int_{x_{0}} W_{k}$ $(s=k, \cdots, n)$ (cf. Lemma 5). Hence there exists a logarithmic monomial $J$ with $\delta_{i}(J)=0(i=0, \cdots, p-1)$ such that $R_{s}\left(z, \dot{X}_{1} z, \cdots, \dot{X}_{n} \cdots \dot{X}_{1} z\right) \prec J \exp \int_{x_{0}} 2 W_{k}$; but $J \exp \int_{x_{0}} 2 W_{k} \prec F \exp \int_{x_{0}} W_{k}$ as a consequence of Lemma $\varepsilon$, proving the assertion.

11. Lemma. Let the hypotheses and notation of Lemma 10 be in force. Then $R_{n}\left(z, \dot{X}_{1} z, \cdots, \dot{X}_{n} \cdots \dot{X}_{1} z\right) \sim-F \exp \int_{x_{0}} W_{k}$ whenever $z \prec 1$.

Proof. $R_{n}\left(z, \dot{X}_{1} z, \cdots, \dot{X}_{n} \cdots \dot{X}_{1} z\right)=P_{n}\left(y, \dot{W}_{1} y, \cdots, \dot{W}_{n} \cdots \dot{W}_{1} y\right) T(n)$ where $T(n) \sim-F \exp \int_{x_{0}} W_{k}$, while by hypothesis $P_{n}\left(y, \dot{W}_{1} y, \cdots, \dot{W}_{n} \cdots \dot{W}_{1} y\right) \sim 1$ whenever $y \prec 1$ and a fortiori whenever $z \prec 1$.

12. LEMMA. Let the hypotheses and notation of Lemma 11 be in force. Let $\left(X_{1}, \cdots, X_{n}\right)$ be an isotone permutation of $\left(X_{1}, \cdots, X_{n}\right)$. Then $S$ is normal with respect to $\left(\bar{X}_{1}, \cdots, \bar{X}_{n}, r\right)$, where $S$ is the operator $\left(-F \exp \int_{x_{0}} W_{k}\right)^{-1} R$.

Proof. Lemmas 8-11 imply $S \sim(w) \dot{X}(n, \cdots, 1)$. Hence $S(0) \prec 1,[H L S]^{\sim}$ $\sim \dot{X}(n, \cdots, 1)$, and $[H L S]^{\sim} \sim \dot{X}^{-}(n, \cdots, 1)$. Let $y_{G}=G K_{1}$.

$$
\begin{aligned}
P\left(y_{g}+y_{G} z\right)=\Sigma\left\{q _ { \hat { i } } \prod _ { t = 0 } ^ { n } \left(\theta^{t} y_{g}\right.\right. & +\left(\theta^{t} y_{G}\right) z+t\left(\theta^{t-1} y_{G}\right) \theta z+\cdots \\
& \left.\left.+t \theta y_{G} \theta^{t-1} z+y_{G} \theta^{t} z\right)^{i_{t}}: i_{0}+\cdots+i_{n} \geqq 2\right\} .
\end{aligned}
$$

Hence $P\left(y_{g}+y_{G} z\right)$ may be written as a sum of terms each having the form

$$
c q_{i} M\left(y_{g}, \theta y_{g}, \cdots, \theta^{n} y_{g} ; y_{G}, \theta y_{G}, \cdots, \theta^{n} y_{G}\right) N\left(z, \theta z, \cdots, \theta^{n} z\right)
$$

where $M$ and $N$ are products of integral powers of their arguments, $c$ is a constant, $M$ is of degree $\geqq 2$, and the differential order of $N$ is $\leqq t^{*}$, where (relative to a fixed $\hat{i}_{\hat{i}} t^{*}$ is the largest integer $t$ such that $i_{t} \neq 0$. Let the differential order of $N$ be $s^{*}$. As in the proofs of the previous lemmas, it is verified that $M \prec M^{*} \exp \int_{x_{0}} 2 W_{k}$ where $M^{*}$ is any sufficiently large logarithmic monomial such that $\delta_{i}\left(M^{*}\right)=0 \quad(i=0, \cdots, p-1)$.

Consider a term for which $s^{*}>k$. Let $B_{i}=-\left(x(\log x) \cdots\left(\log _{r-1} x\right) X_{i}\right)^{-1}$ $(i=1, \cdots, n)$. We have $B_{1} \cdots B_{k} \sim B^{*}$, where $B^{*}$ is a logarithmic monomial with $\delta_{j}\left(B^{*}\right)=0(j=0, \cdots, p-1)$. By the normality of $P$ with respect to $\left(W_{1}, \cdots, W_{n}, r\right)$, $q_{\hat{\imath}} \precsim A_{1} \cdots A_{t^{*}}$ Therefore $A_{1} \cdots A_{t^{*}} M^{*} \exp \int_{x_{0}} 2 W_{k} \succ c q_{\hat{i}} M$; 
and

$$
A_{1} \cdots A_{t^{*}} M^{*} F^{-1} \exp \int_{x_{0}} W_{k} \succ\left(-F \exp \int_{x_{0}} W_{k}\right)^{-1} c q_{\hat{\imath}} M
$$

$$
\begin{aligned}
&\left(-F \exp \int_{x_{0}} W_{k}\right)^{-1} c q_{\hat{\imath}} M\left(B_{1}, \cdots, B_{s^{*}}\right)^{-1} \\
& \precsim \frac{A_{1} \cdots A_{t^{*}}}{B_{k+1} \cdots B_{s^{*}}} \cdot \frac{M^{*}}{B_{1} \cdots B_{k} F} \cdot \exp \int_{x_{0}} W_{k} .
\end{aligned}
$$

Now $\delta_{i}\left(M^{*} / B_{1} \cdots B_{k} F\right)=0(i=0, \cdots, p-1)$; and, since $\bar{X}_{h} \approx W_{h}(h=k+1, \cdots, n)$, we have $B_{h} \approx A_{h}(h=k+1, \cdots, n)$. Hence the right-hand member of (12.1) is $\precsim 1$. Therefore we can express $\left(-F \exp \int_{x_{0}} W_{k}\right)^{-1} \cdot P\left(y_{g}+y_{G} z\right)$ as a sum of terms of the form $f_{\hat{j}^{\prime}} z^{j_{0}}(\theta z)^{j_{1}} \cdots\left(\theta^{n} z\right)^{j_{n}}$ where $j_{s^{*}} f_{\hat{\jmath}} \precsim B_{1} \cdots B_{s^{*}}$ for every $\hat{\jmath}$ such that $s^{*}>k$. The same inequality for $j_{s^{*}} f_{\hat{j}}$ can be verified in very much the same way for terms in which $s^{*} \leqq k$. It follows that $S$ is normal with respect to $\left(X_{1}, \cdots, X_{n}, r\right)$ by $[2, \S 102]$.

13. In this lemma we show that if $P$ is an operator which has been "normalized," i.e., $\sim(r, c, w) \dot{W}(n, \cdots, 1)$ with $P(0)=0$, then the equation $P(y)=0$ has a one-parameter family of solutions $y_{g}+y_{G} z$. These solutions are $\approx H_{1} \exp \int_{x_{0}} W_{k}$, the matching constant $g \neq 0$ being arbitrarily chosen. (For $z \prec 1, y_{g}+y_{G} z$ $\left.\sim y_{g} \sim g H_{1} \exp \int_{x_{0}} W_{k}.\right)$ This is the case for each $k \in(1, \cdots, n)$ such that $[I F]^{\sim}\left(W_{k}, \phi\right)<0$, i.e., such that $\exp \int_{x_{0}} W_{k} \rightarrow 0$ in the direction $\phi$. Thus we have the "expected" number of solutions $\prec 1$ in a weak sense: There are that many separate one-parameter families of solutions $\prec 1$.

Theorem III, below, proceeds beyond this to show how "quasilinear combinations" of such solutions may be formed to obtain a family of solutions $\prec 1$ such that each member of the family corresponds to a simultaneous choice of all available parameters.

LEMMA. Let the hypotheses and notation of Lemma 12 be in force. Then there exists a function $Z \prec 1$ such that $y=y_{g}+y_{G} Z$ satisfies

$$
P\left(y, \dot{W}_{1} y, \cdots, \dot{W}_{n} \cdots \dot{W}_{1} y\right)=0 .
$$

Proof. The related operator $S$ is normal with respect to $\left(\bar{X}_{1}, \cdots, \bar{X}_{n}, r\right)$, so we may invoke Theorem IIIa of [2] to show the existence of $Z \prec 1$ such that $S(Z)=0$. Of course $P\left(y_{g}+y_{G} Z\right)=0$ for such a $Z$, q.e.d.

14. THEOREM III. Let $W_{1} \precsim \ldots \precsim W_{n}$, with each $W_{i} \sim$ a logarithmic monomial in the divergence class. Let $P$ be normal with respect to $\left(W_{1}, \cdots, W_{n}, r\right)$. Let $Y \prec 1$ and let $P(Y)=0$. Let $\left(k_{1}, \cdots, k_{u}\right) \subset(1, \cdots, n)$, and let $[I F] \sim\left(W_{k_{i}}, \phi\right)<0$ $(i=1, \cdots, u)$. Let $\left(W_{1}, \cdots, W_{n}\right)$ be separated. Let $H(k)=\left(1-W_{1}^{-1} W_{k}\right)^{-1} \cdots$ $\left(1-W_{k-1}^{-1} W_{k}\right)^{-1}$. Let $(\gamma, \delta)$ be a subinterval of $(\alpha, \beta)$ in which $[I F] \sim\left(W_{k_{i}}-W_{k_{i+1}}, \theta\right)$ is either always positive or else always negative $(i=1, \cdots, u-1)$. (Such a subinterval exists because of the unblockedness conditions.) Let $W_{1}, \cdots, W_{n}$ be re- 
labeled, if necessary, so that $[I F] \sim\left(W_{k_{i}}-W_{k_{i+1}}, \theta\right)$ is always positive in $(\gamma, \delta)$. (This can be achieved while retaining $W_{1} \precsim \ldots \precsim W_{n}$ and all other relevant structure by virtue of stability under isotone permutations of factorization sequences.) Then there exists a family of functions of one complex variable

$$
\left\{Y^{*}\left(g_{1}, \cdots, g_{u}\right):\left(g_{1}, \cdots, g_{u}\right) \in(\text { complex numbers })^{u}\right\}
$$

such that:

(14.1) The map $\left(g_{1}, \cdots, g_{u}\right) \rightarrow Y^{*}\left(g_{1}, \cdots, g_{u}\right)$ is bijective.

(14.2) For each $(i-1)$-tuple $\left(g_{1}, \cdots, g_{i-1}\right)$ let $\left(g_{1}, \cdots, g_{i-1}, *\right)$ denote the set of all $u$-tuples $\left(h_{1}, \cdots, h_{u}\right)$ such that $h_{j}=g_{j}$ for $j=1, \cdots, i-1(i=1, \cdots, u)$. Then for every $\left(g_{1}, \cdots, g_{i-1}, *\right)$ there exists $W\left(\left(g_{1}, \cdots, g_{i-1}, *\right)\right) \sim W_{k_{i}}$ and $a$ complex number $x\left(\left(g_{1}, \cdots, g_{i-1}, *\right)\right)=x_{0}$ such that $Y^{*}\left(g_{1}, \cdots, g_{i-1}, g_{i}, \cdots, g_{u}\right)-$ $Y^{*}\left(g_{1}, \cdots, g_{i-1}, q_{i}, \cdots, q_{u}\right) \sim\left(g_{i}-q_{i}\right) H\left(k_{i}\right) \exp \int_{x_{0}} W\left(\left(g_{1}, \cdots, g_{i-1}, *\right)\right)$ in $F(\gamma, \delta)$, whenever $g_{i} \neq q_{i}$.

(14.3) For each $\left(g_{1}, \cdots, g_{u}\right), Y^{*}\left(g_{1}, \cdots, g_{u}\right) \prec 1$ in $F(\alpha, \beta)$ and $P\left(Y+Y^{*}\left(g_{1}, \cdots, g_{u}\right)\right)=0$.

Proof. Let $P_{1}(y)=P(Y+y)$. Then, by Lemma $\alpha, P_{1}$ is normal with respect to $\left(W_{1}, \cdots, W_{n}, r\right)$, and it follows that there exist $W_{1}^{\prime}, \cdots, W_{n}^{\prime}$ such that $W_{i}^{\prime} \sim W_{i}$ $(i=1, \cdots, n)$ and such that $P_{1} \sim(r, c, w) \dot{W}^{\prime}(n, \cdots, 1)$ (cf. Lemma 7). For a fixed choice of such functions $W_{i}^{\prime}$ define $W((*))=W_{k_{1}}^{\prime}$; define $x((*))$ as some point belonging to an element of $F(\alpha, \beta)$ in which $W((*))$ is analytic. Then for each nonzero complex number $g_{1}$ define $Y\left(g_{1}\right)$ to be some particular solution of $P_{1}(y)=0$ which is $\sim g_{1} H\left(k_{1}\right) \exp \int_{x_{0}} W((*))$, where $x_{0}=x((*))$, and define $Y(0) \equiv 0$. (The existence of $Y\left(g_{1}\right)$ is guaranteed by Lemma 13.) We now have $P_{1}\left(Y\left(g_{1}\right)\right)=$ $=P\left(Y+Y\left(g_{1}\right)\right)=0$.

Let $g_{1}$ be fixed throughout this paragraph. Let $P_{2}\left(g_{1} ; y\right)=P_{1}\left(Y\left(g_{1}\right)+y\right)$. Then $P_{2}\left(g_{1} ;-\right)$ is normal with respect to $\left(W_{1}, \cdots, W_{n}, r\right)$ by Lemma $\alpha$, and it follows from Lemma 7 that there exist $W_{1}^{\prime \prime}, \cdots, W_{n}^{\prime \prime}$ such that $W_{i}^{\prime \prime} \sim W_{i}(i=1, \cdots, n)$ and such that $P_{2}\left(g_{1} ;-\right) \sim(r, c, w) \dot{W}^{\prime \prime}(n, \cdots, 1)$. For a fixed choice of such $W_{i}^{\prime \prime}$ define $W\left(\left(g_{1}, *\right)\right)=W_{k_{2}}^{\prime \prime}$ and define $x\left(\left(g_{1}, *\right)\right)$ as some point belonging to an element of $F(\alpha, \beta)$ in which $W\left(\left(g_{1}, *\right)\right)$ is analytic. Then for each nonzero complex number $g_{2}$ define $Y\left(g_{1}, g_{2}\right)$ to be some particular solution of $P_{2}\left(g_{1} ; y\right)=0$ which is $\sim g_{2} H\left(k_{2}\right) \exp \int_{x_{0}} W\left(\left(g_{1}, *\right)\right)$, where $x_{0}=x\left(\left(g_{1}, *\right)\right)$, and let $Y\left(g_{1}, 0\right) \equiv 0$. (The existence of $Y\left(g_{1}, g_{2}\right)$ is guaranteed by Lemma 13.) We now have $P_{2}\left(g_{1} ; Y\left(g_{1}, g_{2}\right)\right)=P\left(Y+Y\left(g_{1}\right)+Y\left(g_{1}, g_{2}\right)\right)=$ :0. And so forth: When $W\left(\left(g_{1}, \cdots, g_{s-1}, *\right)\right), x\left(\left(g_{1}, \cdots, g_{s-1}, *\right)\right), P_{s}\left(g_{1}, \cdots, g_{s-1} ;-\right)$, and $Y\left(g_{1}, \cdots, g_{s}\right)$ have been defined for all $\left(g_{1}, \cdots, g_{s}\right)$, we next define

$$
P_{+1}\left(g_{1}, \cdots, g_{s} ; y\right)=P_{s}\left(g_{1}, \cdots, g_{-1} ; Y\left(g_{1}, \cdots, g_{s}\right)+y\right) \text {. }
$$

Then $P_{s+1}\left(g_{1}, \cdots, g_{s} ;-\right)$ is normal with respect to $\left(W_{1}, \cdots, W_{n}, r\right)$ by Lemma $\alpha$, and it follows from Lemma 7 that there exist $W_{1}^{\#}, \cdots, W_{n}^{\#}$ such that $W_{i}^{\#} \sim W_{i}(i=1, \cdots, n)$ and such that $P_{+1}\left(g_{1}, \cdots, g ;-\right) \sim(r, c, w) \dot{W}^{\#}(n, \cdots, 1)$. 
For a fixed choice of such $W_{i}^{\#}$, define $W\left(\left(g_{1}, \cdots, g_{s}, *\right)\right)=W_{k_{s+1}}^{\#}$, and define $x\left(\left(g_{1}, \cdots, g_{s}, *\right)\right)$ as some point belonging to an element of $F(\alpha, \beta)$ in which $W\left(\left(g_{1}, \cdots, g_{s}, *\right)\right)$ is analytic. Then if $g_{s+1} \neq 0$ define $Y\left(g_{1}, \cdots, g_{s+1}\right)$ to be a solution of $P_{s+1}\left(g_{1}, \cdots, g_{s} ; y\right)=0$ which is $\sim g_{s+1} H\left(k_{s+1}\right) \exp \int_{x_{0}} W\left(\left(g_{1}, \cdots, g_{s}, *\right)\right)$, where $x_{0}=x\left(\left(g_{1}, \cdots, g_{s}, *\right)\right)$, and let $Y\left(g_{1}, \cdots, g_{s}, 0\right) \equiv 0$.

For each $u$-tuple $\left(g_{1}, \cdots, g_{u}\right)$ we get $u$ functions in this way: $Y\left(g_{1}\right)$, $Y\left(g_{1}, g_{2}\right), \cdots, Y\left(g_{1}, \cdots, g_{u}\right)$. Define

$$
Y^{*}\left(g_{1}, \cdots, g_{u}\right)=\sum_{v=1}^{u} Y\left(g_{1}, \cdots, g_{v}\right)
$$

Then (14.3) is obviously valid. To verify $(14.2)$, we have $Y^{*}\left(g_{1}, \cdots, g_{u}\right)$ $-Y^{*}\left(g_{1}, \cdots, g_{i-1}, q_{i}, \cdots, q_{u}\right)=A+B-C$, where $A=Y\left(g_{1}, \cdots, g_{i}\right)-Y\left(g_{1}, \cdots, g_{i-1}, q_{i}\right)$, $B=\sum_{v=i+1}^{u} Y\left(g_{1}, \cdots, g_{v}\right)$, and $C=\sum_{v=i+1}^{u} Y\left(g_{1}, \cdots, g_{-1}, q_{i}, \cdots, q_{v}\right)$. Now we have

$$
A \sim\left(g_{i}-q_{i}\right) H\left(k_{i}\right) \exp \int_{x_{0}} W\left(\left(g_{1}, \cdots, g_{i-1}, *\right)\right) \quad\left(x_{0}=x\left(\left(g_{1}, \cdots, g_{i-1}, *\right)\right)\right) .
$$

For the general term in $B$ we have

$$
Y\left(g_{1}, \cdots, g_{v}\right) \sim g_{v} H\left(k_{v}\right) \exp \int_{x_{0}} W\left(\left(g_{1}, \cdots, g_{v-1}, *\right)\right) \quad\left(x_{0}=x\left(\left(g_{1}, \cdots, g_{v-1}, *\right)\right)\right),
$$

and a similar relation for the analogous term in $C(v=1+i, \cdots, u)$. For $v>i$, $H\left(k_{i}\right) / H\left(k_{v}\right) \succsim 1$ in $F(\alpha, \beta)$ and hence in $F(\gamma, \delta)$, while by virtue of the hypotheses we have

$$
\exp \int_{x_{0}} W\left(\left(g_{1}, \cdots, g_{i-1}, *\right)\right) / \exp \int_{x^{\prime} 0} W\left(\left(g_{1}, \cdots, g_{v-1}, *\right)\right)>1
$$

$\left(x_{0}=x\left(\left(g_{1}, \cdots, g_{i-1}, *\right)\right), x^{\prime}{ }_{0}=x\left(\left(g_{1}, \cdots, g_{v-1}, *\right)\right)\right)$ in $F(\gamma, \delta)$. Hence $A \succ B$ in $F(\gamma, \delta)$, and similarly $A \succ C$ in $F(\gamma, \delta)$, so we have $A+B-C \sim A$ in $F(\gamma, \delta)$. This verifies (14.2). (14.1) follows easily from (14.2).

15. With the foregoing notion of a $u$-parameter family we arrive at

THEOREM IV. Let $\alpha, \phi, \beta$ be real numbers with $-\pi \leqq \alpha<\phi<\beta \leqq \pi$. Let $P$ be a differential polynomial with coefficients in an $\overline{L D}(F(\alpha, \beta))$. Let $M$ be a simple principal monomial for $P$, and let $\left(W_{1}, \cdots, W_{n}\right)$ be an asymptotically steady type for $P$ at $M$ which satisfies the separation condition (\$2). Let $\left(W_{k_{1}}, \cdots, W_{k_{u}}\right)$ be the subsequence of $\left(W_{1}, \cdots, W_{n}\right)$ consisting of the $W_{i}$ for which $[I F]^{\sim}\left(W_{i}, \theta\right)<0$ in $(\alpha, \beta)$. Then $P=0$ has a u-parameter family of solutions $Y$ such that $Y \sim M$ in $F(\alpha, \beta)$. I.e., the reduced equation $P(M(1+z))=0$ has a family of solutions $Z \prec 1$ which possess asymptotic developments, in the sense of $\$ 14$, in terms of $u$ asymptotically distinct types of functions of the form $g H \exp \int_{x_{0}} W$.

Proof. This is a corollary of [2, Theorem V] and of Lemma 14 above. 


\section{PART V. APPENDIX}

LEMMA $\alpha$. Let $P^{\prime}$ be normal with respect to $\left(W_{1}, \cdots, W_{n}, r\right)$. Let $Y \prec 1$. Let $P(y)=P^{\prime}(Y+y)$. Then $P$ is normal with respect to $\left(W_{1}, \cdots, W_{n}, r\right)$.

Proof. $[2, \S 91]$ shows that $P \sim(w) \dot{W}(n, \cdots, 1)$. Now by the normality of $P^{\prime}$, $P(y)$ may be expressed as a sum of functions of the form

$$
c q_{\hat{i}} Y^{i_{0}-j_{0}}\left(\theta_{r} Y\right)^{i_{1}-j_{1}} \cdots\left(\theta_{r}^{n} Y\right)^{i_{n}-j_{n}} y^{j_{0}}\left(\theta_{r} y\right)^{j_{1}} \cdots\left(\theta_{r}^{n} y\right)^{j_{n}}
$$

and we must show that $j_{t} q_{\hat{i}} Y^{i_{0}-j_{0}}\left(\theta_{r} Y\right)^{i_{1}-j_{1}} \cdots\left(\theta_{r}^{n} Y\right)^{i_{n}-j_{n}} \succsim A_{1} \cdots A_{t}(t=0, \cdots, n)$, where $A_{i}=-\left(x(\log x) \cdots\left(\log _{r-1} x\right) W_{i}\right)^{-1}$. But this is clear from the fact that $0 \leqq j_{t} \leqq i_{t}, i_{t} q_{\hat{i}} \precsim A_{1} \cdots A_{t}$, and $Y, \theta_{r} Y, \cdots$, and $\theta_{r}^{n} Y$ are $\prec 1$ (cf. $[1, \S 17]$ ).

LEMMA $\beta$. Let $P$ be normal with respect to $\left(W_{1}, \cdots, W_{n}, r\right)$, and let $V_{i} \sim W_{i}(i=1, \cdots, n)$. Then $P$ is normal with respect to $\left(V_{1}, \cdots, V_{n}, r\right)$.

Proof. Since $W_{i} \sim V_{i}$, the relation $P \sim(w) \dot{W}(n, \cdots, 1)$ implies $P \sim(w) \dot{V}(n, \cdots, 1)$. The asymptotic inequalities of $[2, \S 102]$ are readily verified.

LEMMA $\gamma$. Let $W \sim a$ logarithmic monomial in the divergence class in $F(\alpha, \beta)$, with $[I F]^{\sim}(W, \theta)<0$ in $(\alpha, \beta)$. Then $\exp \int_{x_{0}} W \prec 1$ in $F(\alpha, \beta)$.

Proof. If $P(y)=\dot{W} y$, obviously $P \sim(s) \dot{W}(1)$ (with $W_{1}=W$ ). If $x_{1}$ is sufficiently large and $g$ is sufficiently small, the successive approximations of $[2, \S 94]$ can be made to converge to a function $Y$ such that $Y\left(x_{1}\right)=g$ and $Y \prec 1$ in $F(\alpha, \beta)$. From standard uniqueness theory, $Y=g \exp \int_{x_{1}} W$. Since $\exp \int_{x_{0}} W=$ $\left(\exp \int_{x_{0}}^{x_{1}} W\right) \exp \int_{x_{1}} W, \exp \int_{x_{0}} W \prec 1$.

LEMMA $\delta$. Let $W \sim$ a logarithmic monomial in the divergence class and let $G \prec 1$ in $F(\alpha, \beta)$. Let $[I F \sim(W, \theta)$ have no zeros in $(\alpha, \beta)$. Then

(a) if $[I F]^{\sim}(W, \theta)<0$, every solution of $\dot{W} y=G$ is $\prec 1$ in $F(\alpha, \beta)$;

(b) if $[I F]^{\sim}(W, \theta)>0$, just one solution of $W y=G$ is $\prec 1$ in $F(\alpha, \beta)$, and every other solution is $\succ 1$ in $F(\alpha, \beta)$.

Proof. (a) Any solution of $\dot{W} y=G$ has the form

$$
y(x)=g \exp \int_{x_{0}}^{x} W d t-\int_{x_{0}}^{x} W G\left(\exp \int_{t}^{x} W d s\right) d t
$$

for some constant $g$. Clearly all such $y$ are analytic in one and the same element of $F(\alpha, \beta)$, and $y\left(x_{0}\right)=g$. In view of $[2, \S \$ 95-97]$, and standard uniqueness theory, such functions are $\prec 1$ if $g$ is sufficiently small and $x_{0}$ sufficiently large. Applying Lemma $\gamma$ one finds that $y \prec 1$ for any complex $g$ and any relevant $x_{0}$.

(b) $[2, \S \S 95-97]$ show there is one solution $Y_{0}$ which is $\prec 1$. Every other solution is obtained by adding to $Y_{0}$ a multiple of $\exp \int_{x_{0}} W$. But $[I F]^{\sim}(-W, \theta)<0$ wherever $[I F]^{\sim}(W, \theta)>0$, and $\exp \int_{x_{0}} W=\left(\exp \int_{x_{0}}-W\right)^{-1}$; and Lemma $\gamma$ shows 
that $\exp \int_{x_{0}}-W \prec 1$. By $[1, \S 13 \mathrm{~b}], \exp \int_{x_{0}} W \succ 1$, so every solution other than $Y_{0}$ is $\succ 1$.

LEMMA $\varepsilon$. Let $c, \tau$, and $\delta$ be numbers such that $c \neq 0$, $\tau$ is real and positive, and $\delta$ is real. Let $F=\left(\log _{k} x\right)^{-\delta}$, let

$$
W \sim c x^{-1}(\log x)^{-1} \cdots\left(\log _{k-1} x\right)^{-1}\left(\log _{k} x\right)^{-1+\tau}\left(\log _{k+1} x\right)^{a_{1}} \cdots\left(\log _{k+s} x\right)^{a_{s}},
$$

and let $[I F]^{\sim}(W, \theta)<0$ in $(\alpha, \beta)$. Then $F \exp \int_{x_{0}} W \prec 1$ in $F(\alpha, \beta)$.

Proof. $F \exp \int_{x_{0}} W=F\left(x_{0}\right) \exp \int_{x_{0}}(W+D(\log F))$, and the integrand is easily seen to be $\sim W$, so the assertion follows from Lemma $\gamma$.

LEMMA $\zeta$. Let $\left(m_{0}, \cdots, m_{p}\right)$ be a sequence of real numbers different from $(-1, \cdots,-1)$; let $i$ be the least integer such that $m_{i} \neq-1$. Let $N \sim c x^{m_{0}}(\log x)^{m_{1}} \cdots\left(\log _{p} x\right)^{m_{p}}$ in $F(\alpha, \beta)$, where $c$ is a nonzero constant and $-\pi \leqq \alpha<\beta \leqq \pi$. Let $N$ be analytic in $V \in F(\alpha, \beta)$ and let $x_{0} \in V$. Let $M=\left(m_{i}+1\right)^{-1} c\left(\log _{i} x\right)^{m_{i+1}}\left(\log _{i+1} x\right)^{m_{i+1}} \cdots\left(\log _{p} x\right)^{m_{p}}$. Then

(a) $\int_{x_{0}}^{x} N \sim M(x)$ if $N \in$ the divergence class;

(b) if $N \notin$ the divergence class, $\int_{x_{0}} N \precsim 1$ and there exists a unique complex number $A$ such that $A+\int_{x_{0}} N \sim M$.

Proof. $\int_{x_{0}} N$ is a solution of $P(y)=D y-N=0$, an equation to which the entire Strodt theory may be applied. It is easily seen that $M$ is the (unique) principal monomial for $P$. Writing $\int_{x_{0}} N=M(1+Z)$, we see that $Z$ satisfies $Q(Z)=0$, where $Q(z)=z-(-D M / M)^{-1} D z-(N-D M) / D M$. Thus for some complex number $g$, the general solution

$$
z(g, x)=g \exp \int_{x_{0}}^{x}(-D M / M) d t+\int_{x_{0}}^{x}\left(((N-D M) / M) \exp \int_{t}^{x}(-D M / M) d s\right) d t
$$

becomes $Z(x)$. Now $Q(z)=0$ may be written $\dot{W} z=G$, where $W=-D M / M \sim-\left(m_{i}+1\right) x^{-1}(\log x)^{-1} \cdots\left(\log _{i} x\right)^{-1}$, which belongs to the divergence class; and $G=(N-D M) / D M \prec 1$. Furthermore, $[I F]^{\sim}(W, \theta) \equiv-1$ in case (a), and $\equiv+1$ in case (b). By $[2, \S \S 95-97]$, accordingly, every choice of $g$ makes $z(g,-) \prec 1$ in $F(\alpha, \beta)$ in case (a), and just one choice of $g$ makes $z(g,-) \prec 1$ in case (b). In case (b), if $g^{*}$ is that choice, we have $z(g,-)=z\left(g^{*},-\right)+\left(g-g^{*}\right) M\left(x_{0}\right) M^{-1}$. (a) and (b) follow immediately from this and from the relation $\int_{x_{0}} N=M(1+Z)$.

\section{REFERENCES}

1. Walter Strodt, Contributions to the asymptotic theory of ordinary differential equations in the complex domain, Mem. Amer. Math. Soc. No. 13 (1954), 81 pp.

2. - Principal solutions of ordinary differential equations in the complex domain, Mem. Amer. Math. Soc. No. 26 (1957), 107 pp.

COLUMBIA UNIVERSITY,

NeW YORK, NeW YORK 